\title{
Active Diagnosis for Probabilistic Systems
}

\author{
Nathalie Bertrand ${ }^{1}$, Éric Fabre ${ }^{1}$, \\ Stefan Haar ${ }^{1,2}$, Serge Haddad ${ }^{2}$, and Loïc Hélouët ${ }^{1}$ \\ 1 Inria, France \\ ${ }^{2}$ LSV, ENS Cachan \& CNRS \& Inria, France
}

\begin{abstract}
The diagnosis problem amounts to deciding whether some specific "fault" event occurred or not in a system, given the observations collected on a run of this system. This system is then diagnosable if the fault can always be detected, and the active diagnosis problem consists in controlling the system in order to ensure its diagnosability. We consider here a stochastic framework for this problem: once a control is selected, the system becomes a stochastic process. In this setting, the active diagnosis problem consists in deciding whether there exists some observationbased strategy that makes the system diagnosable with probability one. We prove that this problem is EXPTIME-complete, and that the active diagnosis strategies are belief-based. The safe active diagnosis problem is similar, but aims at enforcing diagnosability while preserving a positive probability to non faulty runs, i.e. without enforcing the occurrence of a fault. We prove that this problem requires non belief-based strategies, and that it is undecidable. However, it belongs to NEXPTIME when restricted to belief-based strategies. Our work also refines the decidability/undecidability frontier for verification problems on partially observed Markov decision processes.
\end{abstract}

\section{Introduction}

Diagnosis for discrete event systems was introduced in 11, and can be described as follows: a labeled transition system performs a run, which may contain some specific events called faults. Some of the transition labels are observable, so one gets information about the performed run through its trace, i.e. its sequence of observed labels. The diagnosis problem then amounts to determining whether a fault event occurred or not given the observed trace. The trace is called faulty (resp. correct) if all runs that can have produced it contain (resp. do not contain) a fault. In the remaining cases the trace is called ambiguous. Along with the diagnosis problem comes the diagnosability question: does there exist an infinite ambiguous trace (thus forbidding diagnosis)? For finite transition systems, checking diagnosability was proved to have a polynomial complexity [15].

\footnotetext{
* This work was supported by project ImpRo ANR-2010-BLAN-0317 and the European Union Seventh Framework Programme [FP7/2007-2013] under grant agreement 257462 HYCON2 NOE.
} 
Diagnosis and diagnosability checking have been extended to numerous models (Petri nets [3], pushdown systems [9], etc.) and settings (centralized, decentralized, distributed), and have had an impact on important application areas, e.g. for telecommunication network failure diagnosis. Several contributions have considered enforcing the diagnosability of a system. Under the generic name of active diagnosis, the problems take quite different shapes. They range from the selection of minimal sets of observable labels that make the system diagnosable [4, to the design of controllers that select a diagnosable sublanguage of a system [10], and to online aspects that either turn on and off sensors [4, 13] or modify an action plan [5] in order to reduce the amount of ambiguity. Probabilistic systems have also received some attention [7, 12, with two essential motivations: determining the likelihood of a fault given an observed trace and defining diagnosability for probabilistic systems. Two definitions have been proposed: The A-diagnosability, which requires that the ambiguous traces have a null probability, and the weaker AA-diagnosability, which requires that fault likelihood will converge to one with probability one. Interestingly, the A-diagnosability does not depend on the specific values of transition probabilities, but only on their support: it is thus a structural property of a system, which can be checked in polynomial time on finite state systems.

Here we address the question of active diagnosis for stochastic systems. We elaborate on two recent contributions. The first one [8] improves the work in [10] and designs an observation-based controller that enables a subset of actions in the system in order to make it diagnosable while preserving its liveness. Optimal constructions are then proposed the most relevant for our work being the characterization of unambiguous traces by a deterministic Büchi automaton with minimal size. The second one [1] considers probabilistic Büchi automata, a subclass of partially observed Markov decision processes (POMDP), and proves that checking the existence of strategies that almost surely achieve a Büchi condition on POMDP is EXPTIME-complete. The result was later extended in [2]. This motivates the use of POMDP as semantics for the models we consider.

The first contribution of this paper is a framework for the active diagnosis problem of probabilistic systems. The models we consider are weighted and labeled transition systems, where some transitions represent a fault. Some of the transition labels are observable, and similarly some are controllable. From a given state of the system, and given a set of enabled labels, one derives a transition probability by normalization of transition weights. The active diagnosis problem amounts to designing a label activation strategy that enforces the stochastic diagnosability of the system while preserving its liveness. As a second contribution, this problem is proved to be decidable, and EXPTIME complete. The resulting strategies are belief-based, i.e. they only depend on the set of possible states of the system given past observations, regardless of the exact values of transition weights. As a third contribution, we introduce and analyze the safe active diagnosis problem. It extends the active diagnosis by enforcing a positive probability of correct runs. In other words, this rules out strategies that would reach diagnosability only by enforcing the occurrence of a fault. We prove 
that safe active diagnosis may require non belief-based strategies, and that the existence of such strategies is an undecidable problem. This result refines the decidability/undecidability frontier for POMDP: the existence of a strategy simultaneously ensuring a Büchi condition almost-surely and a safety condition with positive probability is undecidable. This may seem surprising since the existence of strategies for each objective taken separately is decidable. As a last contribution, we prove that, restricted to belief-based strategies, the safe active diagnosis problem becomes decidable and belongs to NEXPTIME.

The paper is organized as follows: section 2 introduces the active diagnosis problem for probabilistic systems, and compares it with the state of the art. Section 3 proposes resolution techniques for active diagnosis. Section 4 analyzes the safe active diagnosis problem. Section 5 concludes this work. A long version of this paper including proofs is available at http://hal.inria.fr/hal-00930919

\section{The Active Diagnosis Problem}

This section recalls diagnosis problems from the literature, and formalizes the new problems we are interested in.

\subsection{Passive (Probabilistic) Diagnosis}

When dealing with stochastic discrete event systems diagnosis, systems are often modeled using labeled transition systems.

Definition 1. A probabilistic labeled transition system $(p L T S)$ is a tuple $\mathcal{A}=$ $\left\langle Q, q_{0}, \Sigma, T, \mathbf{P}\right\rangle$ where:

$-Q$ is a set of states with $q_{0} \in Q$ the initial state;

$-\Sigma$ is a finite set of events;

$-T \subseteq Q \times \Sigma \times Q$ is a set of transitions;

- $\mathbf{P}$ is the transition matrix from $T$ to $\mathbb{Q} \geq 0$ fulfilling for all $q \in Q$ : $\sum_{\left(q, a, q^{\prime}\right) \in T} \mathbf{P}\left[q, a, q^{\prime}\right]=1$.

Observe that a pLTS is a labeled transition system (LTS) equipped with transition probabilities. The transition relation of the underlying LTS is defined by: $q \stackrel{a}{\rightarrow} q^{\prime}$ for $\left(q, a, q^{\prime}\right) \in T$; this transition is then said to be enabled in $q$. A run over the word $\sigma=a_{1} a_{2} \ldots \in \Sigma^{\omega}$ is a sequence of states $\left(q_{i}\right)_{i \geq 0}$ such that

$q_{i} \stackrel{a_{i+1}}{\longrightarrow} q_{i+1}$ for all $i \geq 0$, and we write $q_{0} \stackrel{\sigma}{\Rightarrow}$ if such a run exists. A finite run over $w \in \Sigma^{*}$ is defined analogously, and we write $q \stackrel{w}{\Rightarrow} q^{\prime}$ if such a run ends at state $q^{\prime}$. A state $q$ is reachable if there exists a run $q_{0} \stackrel{w}{\Rightarrow} q$ for some $w \in \Sigma^{*}$. On the other hand, forgetting the labels and merging the transitions with same source and target, one obtains a discrete time Markov chain (DTMC).

Definition 2 (Languages of a pLTS). Let $\mathcal{A}=\left\langle Q, q_{0}, \Sigma, T, \mathbf{P}\right\rangle$ be a $p L T S$. The finite language $\mathcal{L}^{*}(\mathcal{A}) \subseteq \Sigma^{*}$ of $\mathcal{A}$ and the infinite language $\mathcal{L}^{\omega}(\mathcal{A}) \subseteq \Sigma^{\omega}$ of $\mathcal{A}$ are defined by:

$$
\mathcal{L}^{*}(\mathcal{A})=\left\{w \in \Sigma^{*} \mid \exists q: q_{0} \stackrel{w}{\Rightarrow} q\right\} \quad \mathcal{L}^{\omega}(\mathcal{A})=\left\{\sigma \in \Sigma^{\omega} \mid q_{0} \stackrel{\sigma}{\Rightarrow}\right\}
$$


Observations. In order to formalize problems related to diagnosis, we partition $\Sigma$ into two disjoint sets $\Sigma_{o}$ and $\Sigma_{u}$, the sets of observable and of unobservable events, respectively. Moreover, we distinguish a special fault event $f \in \Sigma_{u}$. Let $\sigma$ be a finite word; its length is denoted $|\sigma|$. For $\Sigma^{\prime} \subseteq \Sigma$, define $\mathcal{P}_{\Sigma^{\prime}}(\sigma)$, the projection of $\sigma$ on $\Sigma^{\prime}$, inductively by: $\mathcal{P}_{\Sigma^{\prime}}(\varepsilon)=\varepsilon$; for $a \in \Sigma^{\prime}, \mathcal{P}_{\Sigma^{\prime}}(\sigma a)=$ $\mathcal{P}_{\Sigma^{\prime}}(\sigma) a$; and $\mathcal{P}_{\Sigma^{\prime}}(\sigma a)=\mathcal{P}_{\Sigma^{\prime}}(\sigma)$ for $a \notin \Sigma^{\prime}$. Write $|\sigma|_{\Sigma^{\prime}}$ for $\left|\mathcal{P}_{\Sigma^{\prime}}(\sigma)\right|$, and for $a \in \Sigma$, write $|\sigma|_{a}$ for $|\sigma|_{\{a\}}$. When $\sigma$ is an infinite word, its projection is the limit of the projections of its finite prefixes. This projection can be either finite or infinite. As usual the projection is extended to languages. In the rest of the paper, we will only use $\mathcal{P}_{\Sigma_{o}}$, the projection onto observable events, and hence we will drop the subscript and simply write $\mathcal{P}$ instead of $\mathcal{P}_{\Sigma_{o}}$.

With respect to the partition of $\Sigma=\Sigma_{o} \uplus \Sigma_{u}$, a pLTS $\mathcal{A}$ is convergent if $\mathcal{L}^{\omega}(\mathcal{A}) \cap \Sigma^{*} \Sigma_{u}^{\omega}=\emptyset$ (i.e. there is no infinite sequence of unobservable events from any reachable state). When $\mathcal{A}$ is convergent, then for all $\sigma \in \mathcal{L}^{\omega}(\mathcal{A})$, one has $\mathcal{P}(\sigma) \in \Sigma_{o}^{\omega}$. In the rest of the paper we assume that pLTS are convergent and we will call a sequence a finite or infinite word over $\Sigma$, and an observed sequence a finite or infinite sequence over $\Sigma_{o}$. Clearly, the projection of a sequence on $\Sigma_{o}$ yields an observed sequence. Intuitively, a sequence describes the behavior of a system during an execution, and an observed sequence represents how such a run is perceived. Now, the role of diagnosis is to decide, for any observed sequence, whether a fault has occurred or not.

Ambiguity. A finite (resp. infinite) sequence $\sigma$ is correct if it belongs to $(\Sigma \backslash\{f\})^{*}$ (resp. $\left.(\Sigma \backslash\{f\})^{\omega}\right)$. Otherwise $\sigma$ is called faulty. A correct sequence and a faulty sequence may have the same observed projection, yielding ambiguity.

Definition 3 (Classification of observed sequences). Let $\mathcal{A}$ be a pLTS. An observed sequence $\sigma \in \Sigma_{o}^{\omega}$ is called ambiguous if there exist two sequences $\sigma_{1}, \sigma_{2} \in \mathcal{L}^{\omega}(\mathcal{A})$ such that $\mathcal{P}\left(\sigma_{1}\right)=\mathcal{P}\left(\sigma_{2}\right)=\sigma, \sigma_{1}$ is correct and $\sigma_{2}$ is faulty. An observed sequence $\sigma^{\prime} \in \mathcal{P}\left(\mathcal{L}^{\omega}(\mathcal{A})\right)$ is surely faulty if $\mathcal{P}^{-1}\left(\sigma^{\prime}\right) \cap \mathcal{L}^{\omega}(\mathcal{A}) \subseteq \Sigma^{*} f \Sigma^{\omega}$. An observed sequence $\sigma^{\prime} \in \mathcal{P}\left(\mathcal{L}^{\omega}(\mathcal{A})\right)$ is surely correct if $\mathcal{P}^{-1}\left(\sigma^{\prime}\right) \cap \mathcal{L}^{\omega}(\mathcal{A}) \subseteq$ $(\Sigma \backslash\{f\})^{\omega}$. These notions are defined analogously for finite observed sequences.

Example. Consider the (convergent) pLTS to the left in Fig. 1, where $\Sigma_{u}=$ $\{f, u\}$. We assume uniform distributions so we do not represent the probability matrix $\mathbf{P}$. This pLTS contains infinite ambiguous sequences: immediately after $a$ is observed, an ambiguity appears, and this ambiguity remains in all infinite observed sequences without occurrence of $d$ and finishing with $a b^{\omega}$. Removing the loop at $q_{2}$ and/or $q_{4}$ makes all infinite ambiguous sequences disappear.

In the sequel, we will use the characterization of unambiguous sequences using deterministic Büchi automata [8].

Definition 4 (Büchi automaton). A Büchi automaton over $\Sigma$ is a tuple $\mathcal{B}=$ $\left\langle Q, q_{0}, \Sigma, T, F\right\rangle$ with $\left\langle Q, q_{0}, \Sigma, T\right\rangle$ its underlying LTS and $F \subseteq Q$ an acceptance condition. A run $\left(q_{i}\right)_{i \geq 0}$ is accepting if $q_{i} \in F$ for infinitely many values of $i$. The language $\mathcal{L}(\mathcal{B})$ consists of all words in $\Sigma^{\omega}$ for which there exists an accepting 

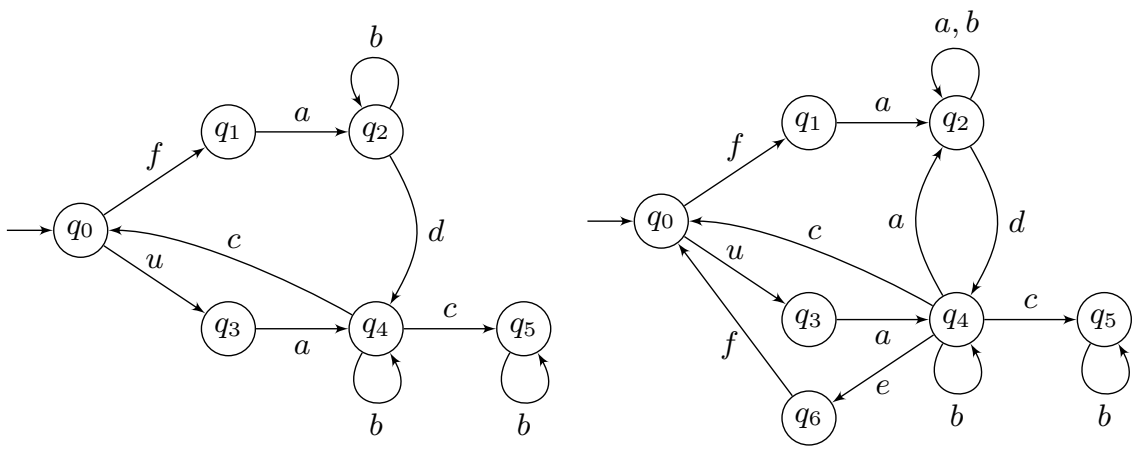

Fig. 1. Two examples of pLTS (cLTS), with $\Sigma_{u}=\{f, u\}$ and $\Sigma_{o}=\{a, b, c, d, e\}$

run. A Büchi automaton is deterministic if for all $q, a,\left\{q^{\prime} \mid q \stackrel{a}{\rightarrow} q^{\prime}\right\}$ is either $a$ singleton or the empty set.

Theorem 1 ([8]). Given a pLTS $\mathcal{A}$ with $n$ states, one can build in exponential time a deterministic Büchi automaton $\mathcal{B}$ with $2^{O(n)}$ states whose language is the set of unambiguous sequences of $\mathcal{A}$.

We briefly sketch the structure of $\mathcal{B}$. Its states are triples $\langle U, V, W\rangle$, where $U, V, W \subseteq Q, U \cup V \cup W \neq \emptyset$ and $V \cap W=\emptyset$, and its transitions are labeled by events from $\Sigma_{o}$, that is $\mathcal{B}$ recognizes observed sequences. The initial state of $\mathcal{B}$ is $\left\langle\left\{q_{0}\right\}, \emptyset, \emptyset\right\rangle$. Given an observed sequence $\sigma$ reaching state $\langle U, V, W\rangle, U$ is the set of states of $\mathcal{A}$ reached by a correct sequence with projection $\sigma$, and $V \cup W$ is the set of states of $\mathcal{A}$ reached by a faulty sequence with projection $\sigma$. When $U=\emptyset, \sigma$ is the projection of faulty sequences of $\mathcal{A}$. The decomposition between $V$ and $W$ reflects the fact that $\mathcal{B}$ tries to "solve the ambiguity" between $U$ and $W$ (when both are non empty), while $V$ corresponds to a waiting room of states reached by faulty sequences that will be examined when the current ambiguity is resolved. Given some new observation $a$, a transition from $\langle U, V, W\rangle$ to the new state $\left\langle U^{\prime}, V^{\prime}, W^{\prime}\right\rangle$ is defined as follows. $U^{\prime}$ is the set of states reached from $U$ by a correct sequence with projection $a$. Let $Y$ be the set of states reached from $U$ by a faulty sequence with projection $a$, or reached from $V$ by a sequence with projection $a$. When $W$ is non empty then $W^{\prime}$ is the set of states reached from $W$ by a sequence with projection $a$ and $V^{\prime}=Y$. Otherwise, the faulty sequences ending in states memorized by $W$ cannot be extended by a sequences with projection $a$, and we set $V^{\prime}=\emptyset$ and $W^{\prime}=Y$. The ambiguity between $U$ and $W$ has been resolved, but new ambiguity may arise between $U^{\prime}$ and $W^{\prime}$. Accepting states in $F$ are triples $\langle U, V, W\rangle$ with $U=\emptyset$ or $W=\emptyset$. Hence, all infinite observed sequence of $\mathcal{A}$ passing infinitely often through $F$ are not ambiguous (they resolve ambiguities one after another) and are accepted by $\mathcal{B}$.

We are now in position to define diagnosability. It is well-known that given a pLTS $\mathcal{A}$ and a Büchi automaton $\mathcal{B}$, the set of sequences of $\mathcal{A}$ accepted by $\mathcal{B}$ is measurable [14. So the following definition is sound. 
Definition 5 (Diagnosability). A $p L T S \mathcal{A}$ is diagnosable if the set of sequences yielding ambiguous observed sequences has null measure.

It is safely diagnosable if it is diagnosable and the set of correct sequences has positive measure.

The notion of a safely diagnosable pLTS is introduced to ensure that fault occurrence is not almost sure. This property is important: a diagnosable system which is not safely diagnosable contains only faulty infinite runs. In the rest of the paper, we will consider active diagnosis, that is, ways to force a system to become diagnosable using a controller. If a controlled system is not safely diagnosable, then the diagnosis solution enforced by the controller is not acceptable.

Example. Consider again the pLTS to the left in Fig. 1. The only ambiguous observed (infinite) sequences necessarily terminate with $a b^{\omega}$. But the probability to produce such a sequence is null, as the system will reach $q_{5}$ with probability one. In other words, ambiguity vanishes at the first occurrence of $d$ or $c b$. Since $c b$ occurs with probability one, this pLTS is diagnosable. This pLTS is also safely diagnosable, as it can produce correct sequences with a positive probability: there is a positive probability to reach $q_{5}$ by sequence $u a c$. If one removes state $q_{5}$ and its connected transitions, the system remains diagnosable, but is not safely diagnosable anymore: as the graph of the pLTS is strongly connected, every transition will be visited (infinitely often) with probability 1 implying that $f$ occurs.

\subsection{Active Probabilistic Diagnosis}

In order to allow control over the actions of a system while preserving the possibility of a probabilistic semantic, we introduce controllable weighted labelled transition system where probabilities are replaced by weights.

Definition 6. A controllable weighted labelled transition system (cLTS) is a tuple $\mathcal{C}=\left\langle Q, q_{0}, \Sigma, T\right\rangle$ where:

$-Q$ is a finite set of states with $q_{0} \in Q$ the initial state;

- the event alphabet $\Sigma$ is partitionned into observable $\Sigma_{o}$ and unobservable $\Sigma_{u}$ events, and also partitionned into controllable $\Sigma_{c}$ and uncontrollable $\Sigma_{e}(e$ for environment) events;

$-\Sigma_{u}=\{f, u\}$ contains a faulty event, and a non-faulty one;

$-T: S \times \Sigma \times S \rightarrow \mathbb{N}$ is the transition function, labelling transitions with integer weights.

A cLTS has an underlying LTS where the transition relation is defined by $q \stackrel{a}{\rightarrow}$ $q^{\prime}$ if $T\left(q, a, q^{\prime}\right)>0$. All previous definitions that do not depend on probabilities equally apply to cLTS. We denote by $\operatorname{Ena}(q)$ the set of events that are enabled in $q$ : $\operatorname{Ena}(q)=\left\{a \in \Sigma \mid \exists q^{\prime}, T\left(q, a, q^{\prime}\right)>0\right\}$. We assume that the cLTS is convergent and live: for all $q$, $\operatorname{Ena}(q) \neq \emptyset$. 
Let $\mathcal{C}=\left\langle Q, q_{0}, \Sigma, T\right\rangle$ be a cLTS. For $q \in Q$ and $\Sigma^{\bullet} \subseteq \Sigma$, we define

$$
G^{\Sigma^{\bullet}}(q)=\sum_{a \in \Sigma^{\bullet}, q^{\prime} \in Q} T\left(q, a, q^{\prime}\right)
$$

as the (possibly null) global outgoing weight from $q$ restricted to $\Sigma^{\bullet}$-events. Similarly, we define a normalization of the transition relation restricted to $\Sigma^{\bullet}$ by

$$
T^{\Sigma^{\bullet}}\left(q, a, q^{\prime}\right)=\left\{\begin{array}{l}
\frac{T\left(q, a, q^{\prime}\right)}{G^{\bullet}(q)} \text { if } a \in \Sigma^{\bullet} \text { and } T\left(q, a, q^{\prime}\right)>0 \\
0 \text { otherwise }
\end{array}\right.
$$

For a given finite set $X$, we define by $\operatorname{Dist}(\mathrm{X})$ the set of probabilistic distributions over $X$. Let $x \in X$, we denote by $\mathbf{1}_{x}$ the Dirac distribution on $x$. For a distribution $\delta \in \operatorname{Dist}(\mathrm{X})$, the support of $\delta$ is the set $\operatorname{Supp}(\delta)=\{x \in X \mid \delta(x)>0\}$.

A strategy for a cLTS $\mathcal{C}$ is a mapping $\pi: \Sigma_{o}^{*} \rightarrow \operatorname{Dist}\left(2^{\Sigma}\right)$ such that for every $\sigma \in \Sigma_{o}^{*}$, for every $\Sigma^{\prime} \in \operatorname{Supp}(\pi(\sigma)), \Sigma^{\prime} \supseteq \Sigma_{e}$. A strategy consists in, given some observation, randomly choosing a subset of allowed events that includes the uncontrollable events. Given a cLTS $\mathcal{C}$ and a strategy $\pi$, we consider configurations of the form $\left(\sigma, q, \Sigma^{\bullet}\right) \in \Sigma_{o}^{*} \times Q \times 2^{\Sigma}$ where $\sigma$ is the observed sequence, $q$ is the current state and $\Sigma^{\bullet}$ is a set of events allowed by $\pi$ after observing $\sigma$. We define inductively the set $\operatorname{Reach}_{\pi}(\mathcal{C})$ of reachable configurations under $\pi$ :

- for all $\Sigma^{\bullet} \in \operatorname{Supp}(\pi(\varepsilon)),\left(\varepsilon, q_{0}, \Sigma^{\bullet}\right) \in \operatorname{Reach}_{\pi}(\mathcal{C})$;

- for all $\left(\sigma, q, \Sigma^{\bullet}\right) \in \operatorname{Reach}_{\pi}(\mathcal{C})$, for all $a \in \Sigma_{u} \cap \Sigma^{\bullet}$, such that $q \stackrel{a}{\rightarrow} q^{\prime}$ $\left(\sigma, q^{\prime}, \Sigma^{\bullet}\right) \in \operatorname{Reach}_{\pi}(\mathcal{C})$, denoted $\left(\sigma, q, \Sigma^{\bullet}\right) \stackrel{a}{\rightarrow}_{\pi}\left(\sigma, q^{\prime}, \Sigma^{\bullet}\right)$;

- for all $\left(\sigma, q, \Sigma^{\bullet}\right) \in \operatorname{Reach}_{\pi}(\mathcal{C})$, for all $a \in \Sigma_{o} \cap \Sigma^{\bullet}$ such that $q \stackrel{a}{\rightarrow} q^{\prime}$ and $\Sigma^{\bullet \prime} \in \operatorname{Supp}(\pi(\sigma a)),\left(\sigma a, q^{\prime}, \Sigma^{\bullet \prime}\right) \in \operatorname{Reach}_{\pi}(\mathcal{C})$,

denoted $\left(\sigma, q, \Sigma^{\bullet}\right) \stackrel{a}{\rightarrow} \pi\left(\sigma a, q^{\prime}, \Sigma^{\bullet \prime}\right)$.

A strategy $\pi$ is said to be live if for every configuration $\left(\sigma, q, \Sigma^{\bullet}\right) \in \operatorname{Reach}_{\pi}(\mathcal{C})$, $G^{\Sigma^{\bullet}}(q) \neq 0$. Live strategies are the only relevant strategies as the other strategies introduce deadlocks. We are now in position to introduce the semantics of a cLTS. It is defined w.r.t. to some live strategy $\pi$ as a pLTS. Its set of states is $\operatorname{Reach}_{\pi}(\mathcal{C})$ with an initial state whose goal is to randomly select w.r.t. $\pi$ the initial control. The transition probabilities are defined by $T^{\Sigma^{\bullet}}$ accordingly to the current control $\Sigma^{\bullet}$ except that when an observable action occurs it must be combined with the random choice (w.r.t. $\pi$ ) of the next control.

Definition 7. Let $\mathcal{C}$ be a CLTS and $\pi$ be a live strategy, the $p L T S \mathcal{C}_{\pi}$ induced by strategy $\pi$ on $\mathcal{C}$ is defined as $\mathcal{C}_{\pi}=\left\langle Q_{\pi}, \Sigma, q_{0 \pi}, T_{\pi}, \mathbf{P}_{\pi}\right\rangle$ where:

$-Q_{\pi}=\left\{q_{0 \pi}\right\} \cup \operatorname{Reach}_{\pi}(\mathcal{C})$;

- for all $\left(\varepsilon, q_{0}, \Sigma^{\bullet}\right) \in \operatorname{Reach}_{\pi}(\mathcal{C}),\left(q_{0 \pi}, u,\left(\varepsilon, q_{0}, \Sigma^{\bullet}\right)\right) \in T_{\pi}$;

- for all $\left(\sigma, q, \Sigma^{\bullet}\right),\left(\sigma^{\prime}, q^{\prime}, \Sigma^{\bullet \prime}\right) \in \operatorname{Reach}_{\pi}(\mathcal{C})$, $\left(\left(\sigma, q, \Sigma^{\bullet}\right), a,\left(\sigma^{\prime}, q^{\prime}, \Sigma^{\bullet \prime}\right)\right) \in T_{\pi}$ iff $\left(\sigma, q, \Sigma^{\bullet}\right) \stackrel{a}{\rightarrow}_{\pi}\left(\sigma^{\prime}, q^{\prime}, \Sigma^{\bullet \prime}\right)$;

- for all $\left(\varepsilon, q_{0}, \Sigma^{\bullet}\right) \in \operatorname{Reach}_{\pi}(\mathcal{C}), \mathbf{P}_{\pi}\left(q_{0 \pi}, u,\left(\varepsilon, q_{0}, \Sigma^{\bullet}\right)\right)=\pi(\varepsilon)\left(\Sigma^{\bullet}\right)$;

- for all $\left(\left(\sigma, q, \Sigma^{\bullet}\right), a,\left(\sigma, q^{\prime}, \Sigma^{\bullet}\right)\right) \in T_{\pi}$, for all $a \in \Sigma_{u} \cap \Sigma^{\bullet}$, $\mathbf{P}_{\pi}\left(\left(\sigma, q, \Sigma^{\bullet}\right), a,\left(\sigma, q^{\prime}, \Sigma^{\bullet}\right)\right)=T^{\Sigma^{\bullet}}\left(q, a, q^{\prime}\right)$; 


$$
\begin{gathered}
- \text { for all }\left(\left(\sigma, q, \Sigma^{\bullet}\right), a,\left(\sigma a, q^{\prime}, \Sigma^{\bullet^{\prime}}\right)\right) \in T_{\pi}, \text { for all } a \in \Sigma_{o} \cap \Sigma^{\bullet}, \\
\mathbf{P}_{\pi}\left(\left(\sigma, q, \Sigma^{\bullet}\right), a,\left(\sigma a, q^{\prime}, \Sigma^{\bullet^{\prime}}\right)\right)=T^{\Sigma^{\bullet}}\left(q, a, q^{\prime}\right) \cdot \pi(\sigma . a)\left(\Sigma^{\bullet \prime}\right) .
\end{gathered}
$$

We can now formalize the decision problems we are interested in.

Definition 8 ((Safe) Active probabilistic diagnosis). Given a cLTS $\mathcal{C}=$ $\left\langle Q, q_{0}, \Sigma, T\right\rangle$, the active probabilistic diagnosis problem asks, whether there exists a live strategy $\pi$ in $\mathcal{C}$ such that the $p L T S \mathcal{C}_{\pi}$ is diagnosable. The safe active probabilistic diagnosis problem asks whether there exists a live strategy $\pi$ in $\mathcal{C}$ such that the pLTS $\mathcal{C}_{\pi}$ is safely diagnosable. The synthesis problems consists in building a live strategy $\pi$ in $\mathcal{C}$ such that the $p L T S \mathcal{C}_{\pi}$ is (safely) diagnosable.

Example. Consider the cLTS to the right in Fig. 11 with all weights equal to 1 and $\Sigma_{o}=\Sigma_{c}$. Without control, the system is not diagnosable as the observed sequence $a a d c b^{\omega}$ is ambiguous, and it has a positive probability. So the strategy should disable action $a$ for each correct observed sequence ending by $a b^{*}$. In addition, if this strategy always forbids $c$, the system becomes diagnosable, but the occurrence of a fault is enforced: so it is not safely diagnosable. Alternatively, if the strategy always forbids $e$, the system becomes safely diagnosable, as we obtain a pLTS "weakly probabilistically bisimilar" to the one on the left in Fig.1

\section{Analysis of the Active Probabilistic Diagnosis Problem}

To solve the active probabilistic diagnosis problem, we reduce it to a decidable problem on POMDP: namely, the existence of a strategy ensuring a Büchi objective with probability one [1,2].

Definition 9 (POMDP). A partially observable Markov decision process $(P O M D P)$ is a tuple $\mathrm{M}=\left\langle Q, q_{0}\right.$, Obs, Act, $\left.T\right\rangle$ where

- $Q$ is a finite set of states with $q_{0}$ the initial state;

- Obs : $Q \rightarrow \mathcal{O}$ assigns an observation $\mathrm{O} \in \mathcal{O}$ to each state.

- Act is a finite set of actions;

$-T: Q \times \operatorname{Act} \rightarrow \operatorname{Dist}(\mathrm{Q})$ is a partial transition function. Letting $\operatorname{Ena}(q)=\{a \in$ Act $\mid T(q, a)$ is defined $\}$, we assume that:

- for all $q \in Q$, $\operatorname{Ena}(q) \neq \emptyset$, and

- whenever $\operatorname{Obs}(q)=\operatorname{Obs}\left(q^{\prime}\right)=\mathrm{O}$, then $\operatorname{Ena}(q)=\operatorname{Ena}\left(q^{\prime}\right)$ and slightly abusing our notation, we will denote by $\mathrm{Ena}(\mathrm{O})$ the set of events enabled in every state with observation $\mathrm{O}$.

A decision rule is an item of Dist(Act) that resolves non-determinism by randomization. A strategy maps histories of observations to decision rules. Formally, a strategy is a function $\pi: \mathcal{O}^{+} \rightarrow \operatorname{Dist}\left(\right.$ Act) such that for all $\mathrm{O}_{1} \cdots \mathrm{O}_{i}$, $\operatorname{Supp}\left(\pi\left(\mathrm{O}_{1} \cdots \mathrm{O}_{i}\right)\right) \subseteq \operatorname{Ena}\left(O_{i}\right)$. Given a strategy $\pi$ and an initial distribution $\delta$ over states, a POMDP M becomes a stochastic process that can be represented 
by a possibly infinite pLTS denoted $\mathrm{M}(\pi)$. One denotes $\mathbb{P}_{\pi}^{\delta}(\mathrm{Ev})$ the probability that event Ev is realized in this process.

A belief is a subset of $\mathrm{Obs}^{-1}(\mathrm{O})$ for some observation $\mathrm{O}$ that corresponds to the possible reachable states w.r.t. some sequence of observations. The initial belief is $\left\{q_{0}\right\}$ and given a current belief $B$, a decision rule $\delta$ and a observation $O$, the belief $\Delta(B,(\delta, \mathrm{O}))$ obtained after $\delta$ has been applied and $\mathrm{O}$ has been observed is defined by: $\bigcup_{q \in B, a \in \operatorname{Supp}(\delta)} \operatorname{Supp}(T(q, a)) \cap \mathrm{Obs}^{-1}(\mathrm{O})$. A strategy which only depends on the current belief is called a belief-based strategy.

In order to provide a POMDP $\mathrm{M}_{\mathcal{C}}$ for the diagnosis problems of a cLTS $\mathcal{C}$, we face several difficulties. First, in a cLTS the observations are related to actions while in a POMDP they are related to states. Fortunately all the information related to ambiguity is included in the deterministic Büchi automaton described in section 2. Thus (with one exception) the states are pairs of a state of the Büchi automaton and a state of the cLTS. In $\mathcal{C}$, the control is performed by allowing a subset of events. Thus actions of $\mathrm{M}_{\mathcal{C}}$ are subset of events that includes the uncontrollable events. Given some control $\Sigma^{\prime}$, for defining the transition probability of $\mathrm{M}_{\mathcal{C}}$ from $(l, q)$ to $\left(l^{\prime}, q^{\prime}\right)$, one must consider all paths in $\mathcal{C}$ labelled by events of $\Sigma^{\prime}$ from $q$ to $q^{\prime}$ such that the last event (say $b$ ) is the single observable one. The probability of any such path is obtained by the product of the individual step probabilities. The latter are then defined by the normalization of weights w.r.t. $\Sigma^{\prime}$. They cannot be infinite paths of unobservable events due to the convergence of $\mathcal{C}$. However some path can reach a state where no event of $\Sigma^{\prime}$ is possible. In other words, the control $\Sigma^{\prime}$ applied in $(l, q)$ has a non null probability to reach a deadlock (i.e. the chosen decision rule leads to a non live strategy for the cLTS). In order to capture this behaviour and to obtain a non defective probability distribution, we add an additional state lost, that corresponds to such deadlocks. The next definition formalizes our approach.

Definition 10. The POMDP $\mathrm{M}_{\mathcal{C}}=\left\langle Q^{\mathrm{M}}, q_{0}^{\mathrm{M}}\right.$, Obs, Act, $\left.T^{\mathrm{M}}\right\rangle$ derived from a $c L T S \mathcal{C}=\left\langle Q, q_{0}, \Sigma, T\right\rangle$ and its associated deterministic Büchi automaton $\mathcal{B}=$ $\left\langle L, l_{0}, \Sigma_{o}, T^{\mathcal{B}}, F\right\rangle$ is defined by:

- $Q^{\mathrm{M}}=L \times Q \uplus\{$ lost $\}$ with $q_{0}^{\mathrm{M}}=\left(\left(l_{0}, q_{0}\right)\right.$;

- the set of observations is $\mathcal{O}=L \cup\{$ lost $\}$, with $\operatorname{Obs}((l, q))=l$ and $\operatorname{Obs}($ lost $)=$ lost;

- Act $=\left\{\Sigma^{\prime} \mid \Sigma^{\prime} \supseteq \Sigma_{e}\right\}$;

- for all $(l, q) \in Q^{\mathrm{M}}$ and $\Sigma^{\prime} \in$ Act, $T^{\mathrm{M}}\left((l, q), \Sigma^{\prime}\right)=\mu$ where:

- $\mu\left(\left(l^{\prime}, q^{\prime}\right)\right)$ is defined by (with $q_{n}=q$ when $\left.n=0\right)$ :

$$
\sum_{\substack{l \stackrel{b}{\rightarrow} l^{\prime} \\ b \in \Sigma^{\prime} \cap \Sigma_{o}}} \sum_{\substack{l^{a_{1}} \rightarrow \\ a_{1} \cdots a_{n} \in \Sigma^{\prime} \cap \Sigma_{u}}} T^{\Sigma^{\prime}}\left(q, a_{1}, q_{1}\right) \cdot\left(\prod_{i=1}^{n-1} T^{\Sigma^{\prime}}\left(q_{i}, a_{i+1}, q_{i+1}\right)\right) \cdot T^{\Sigma^{\prime}}\left(q_{n}, b, q^{\prime}\right)
$$


- $\mu$ (lost) is defined by:

$$
\begin{gathered}
\sum_{\substack{q \stackrel{a_{1}}{q} q_{1} \ldots \frac{a_{n}}{a_{1} \cdots a_{n} \in \Sigma^{\prime} \cap \Sigma_{u}} \\
G^{\Sigma^{\prime}}\left(q_{n}\right)=0}} T^{\Sigma^{\prime}}\left(q, a_{1}, q_{1}\right) \cdot \prod_{i=1}^{n-1} T^{\Sigma^{\prime}}\left(q_{i}, a_{i+1}, q_{i+1}\right) \\
-T^{\mathrm{M}}\left(\text { lost }, \Sigma^{\prime}\right)=\mathbf{1}_{\text {lost }} \text { for all } \Sigma^{\prime} \in \text { Act. }
\end{gathered}
$$

Given $\mathcal{C}$, the construction of the Büchi automaton $\mathcal{B}$ is performed in exponential time. The construction of $\mathrm{M}_{\mathcal{C}}$ is also done in exponential time. Indeed, there is an exponential blowup for Act but again w.r.t. $\mathcal{C}$. Finally, while the distributions $\mu$ of action effects are presented in the definition as sums over paths of $\mathcal{C}$, each one can be computed by a matrix inversion in polynomial time (as done in discrete time Markov chains).

The next lemma is a straightforward consequence of the properties of $\mathcal{B}$ and the above definition of $M_{\mathcal{C}}$. Here we use LTL notations to denote sets of paths in a POMDP, such as $\diamond, \square$ and $\square \diamond$ for eventually, always and infinitely often respectively.

Lemma 1. $\mathcal{C}$ is actively diagnosable if and only if there exists a strategy $\pi$ in $\mathrm{M}_{\mathcal{C}}$ such that $\mathbb{P}_{\pi}^{q_{0}}\left(\mathrm{M}_{\mathcal{C}} \models \square \diamond(W=\emptyset \vee U=\emptyset)\right)=1$.

Moreover, $\mathcal{C}$ is safely actively diagnosable if and only if there exists a strategy $\pi$ in $\mathrm{M}_{\mathcal{C}}$ such that $\mathbb{P}_{\pi}^{q_{0}}\left(\mathrm{M}_{\mathcal{C}}=\square \diamond(W=\emptyset \vee U=\emptyset)\right)=1$ and $\mathbb{P}_{\pi}^{q_{0}}\left(\mathrm{M}_{\mathcal{C}} \models \square(U \neq\right.$ $\emptyset))>0$.

In the statement of Lemma 1. $W=\emptyset \vee U=\emptyset$ is a shorthand to denote the set of states $(\langle U, V, W\rangle, q)$ in $\mathrm{M}_{\mathcal{C}}$ such that either $W=\emptyset$ or $U=\emptyset$; similarly, $U \neq \emptyset$ represents the set of states $(\langle U, V, W\rangle, q)$ such that $U \neq \emptyset$. As a consequence of Lemma 1, the active diagnosis problem for controllable LTS reduces to the existence of an almost-sure winning strategy for a Büchi objective on some exponential size POMDP.

Theorem 2. The active probabilistic diagnosis decision and synthesis problems are EXPTIME-complete. There exists a family $\left(\mathcal{C}_{n}\right)_{n \in \mathbb{N}}$ of actively diagnosable cLTS with the size of $\mathcal{C}_{n}$ in $O(n)$, and such that any winning strategy for $\mathrm{M}_{\mathcal{C}_{n}}$ diagnosable requires at least $2^{\Omega(n)}$ memory-states.

The EXPTIME upper bound may seem surprising, since $M_{\mathcal{C}}$ is exponential in the size of $\mathcal{C}$, and the procedure to decide whether there exists a strategy in a POMDP to ensure a Büchi objective with probability 1 is in EXPTIME, due to the use of beliefs. However, in the POMDP $\mathrm{M}_{\mathcal{C}}$ we consider, the information on the belief is already contained in the state $(\langle U, V, W\rangle, q)$, as $U \cup V \cup W$. Therfore, a second exponential blowup, due to the beliefs, is avoided and the active probabilistic diagnosis problem remains in EXPTIME. 


\section{Analysis of the Safe Active Probabilistic Diagnosis Problem}

As will be shown below, the status of the active diagnosis problem changes when the safety requirement is added. The next proposition highlights this difference and it is the basis for the undecidability result of Theorem 3 .

Proposition 1. There exists a cLTS which is safely actively diagnosable and such that all belief-based strategies are losing.

Proof. Let us consider the cLTS of Figure 2 with $\Sigma_{u}=\{u, f\}$ and $\Sigma_{e}=\{u, f, c\}$, and where all weights are equal to 1.

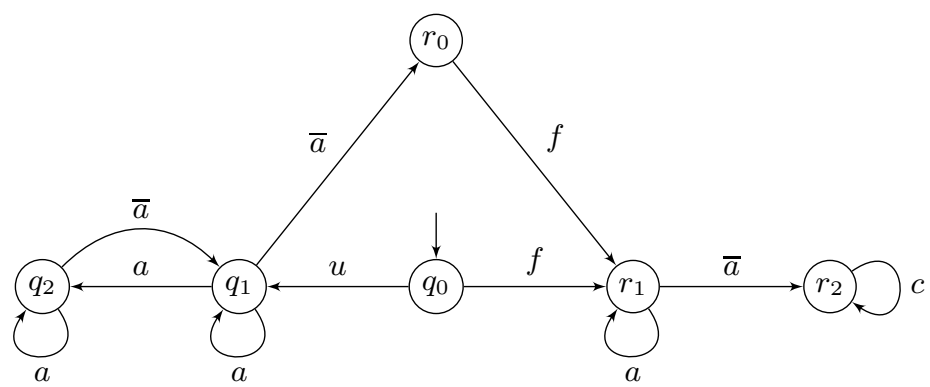

Fig. 2. A cLTS with only non belief-based strategies for safe diagnosis

Pick any sequence of positive integers $\left\{\alpha_{i}\right\}_{i \geq 1}$ such that $\prod_{i \geq 1} 1-2^{-\alpha_{i}}>0$. Define $A=\{a\} \cup \Sigma_{e}$ and $\bar{A}=\{\bar{a}\} \cup \Sigma_{e}$. We claim that the strategy $\pi$ that consists in selecting, after $n$ observations, the $n^{\text {th }}$ subset in the following sequence $A^{\alpha_{1}} \bar{A} A^{\alpha_{2}} \bar{A} \ldots$, is winning. Observe that after an observable sequence of length $i \leq \alpha_{1}$, the system is either after a faulty sequence in $r_{1}$ with probability $\frac{1}{2}$, or after a correct sequence in $q_{1}$ with probability $2^{-i-1}$, or after a correct sequence in $q_{2}$ with probability $\frac{1}{2}\left(1-2^{-i}\right)$. So, after an observable sequence of length $\alpha_{1}+1$, the system is either after a faulty sequence in $r_{2}$ with probability $\frac{1}{2}$, or after a faulty sequence in $r_{1}$ (via $\left.r_{0}\right)$ with probability $2^{-\alpha_{1}-1}$, or after a correct sequence in $q_{1}$ with probability $\frac{1}{2}\left(1-2^{-\alpha_{1}}\right)$. At the next step, the faulty sequence in $r_{2}$ is then detected by the occurrence of $c$.

Iterating this process we conclude that:

- any fault that may occur after $\pi$ is applied up to $A^{\alpha_{1}} \bar{A} A^{\alpha_{2}} \bar{A} \ldots A^{\alpha_{i}} \bar{A}$, is detected after $\pi$ is applied up to $A^{\alpha_{1}} \bar{A} A^{\alpha_{2}} \bar{A} \ldots A^{\alpha_{i+1}} \bar{A} A$. So the (full) strategy $\pi=A^{\alpha_{1}} \bar{A} A^{\alpha_{2}} \bar{A} \ldots$ surely detects faults.

- the probability that there is an infinite correct sequence is equal to $\frac{1}{2} \prod_{i \geq 1} 1-$ $2^{-\alpha_{i}}>0$, due to our choice of the $\alpha_{i}$ 's. Therefore, correct sequences have positive probability under $\pi$. 
Consider a belief-based strategy $\pi$. There are three possible subsets of allowed events: $A, \bar{A}$ and $\Sigma$. The decision rule associated with belief $\left\{q_{0}\right\}$ must allow $a$ in order to get the possibility of a correct sequence which, in case $a$ occurs, leads to belief $\left\{q_{1}, q_{2}, r_{1}\right\}$. We should clarify here that beliefs do not correspond to the possible current states. They represent the possible states after the last observed event. For instance, when the belief is $\left\{q_{0}\right\}$, the current state may either be $q_{0}$, or $q_{1}$ after action $u$, or $r_{1}$ after fault $f$. Consider the (randomized) decision rule of $\pi$ associated with belief $\left\{q_{1}, q_{2}, r_{1}\right\}: p_{A} \cdot A+p_{\bar{A}} \cdot \bar{A}+p_{\Sigma} \cdot \Sigma$ (denoted $\left.\boldsymbol{p}\right)$. If $p_{A}=1$, then the possible first fault remains undetected, and $\pi$ is losing. So $\bar{a}$ may occur leading to belief $\left\{q_{1}, r_{0}, r_{2}\right\}$.

Consider the decision rule of $\pi$ associated with belief $\left\{q_{1}, r_{0}, r_{2}\right\}: p_{A}^{\prime} \cdot A+p_{\bar{A}}^{\prime}$. $\bar{A}+p_{\Sigma}^{\prime} \cdot \Sigma\left(\right.$ denoted $\left.\boldsymbol{p}^{\prime}\right)$. If $p_{\bar{A}}^{\prime}=1$, then at the next instant, there is no possible correct sequence, and $\pi$ is losing.

So $p_{\frac{1}{A}}^{\prime}<1$ and $p_{A}<1$. Assume now that the current distribution of states is $\alpha q_{1}+\beta r_{0}+(1-\alpha-\beta) r_{2}$ (with belief $\left.\left\{q_{1}, r_{0}, r_{2}\right\}\right)$. The distribution after the next occurrence of $\bar{a}$ is defined by $\alpha_{\boldsymbol{p}, \boldsymbol{p}^{\prime}} \alpha q_{1}+\left(1-\alpha \boldsymbol{p}_{\boldsymbol{p}, \boldsymbol{p}^{\prime}}\right) \alpha r_{0}+(1-\alpha) r_{2}$, where $\alpha_{\boldsymbol{p}, \boldsymbol{p}^{\prime}}<1$ only depends on $\boldsymbol{p}$ and $\boldsymbol{p}^{\prime}$. A correct sequence implies an infinite number of $\bar{a}$; after $n$ occurrences of $\bar{a}$ the probability of a correct sequence is bounded by $\alpha_{\boldsymbol{p}, \boldsymbol{p}^{\prime}}^{n}$. So the probability of an infinite correct sequence is null, and $\pi$ is losing.

Theorem 3. The safe active diagnosis problem for cLTS is undecidable.

Proof (sketch). We perform a reduction from the following undecidable problem: given a blind POMDP and a set $F$ of states, does there exist a strategy that ensures the Büchi objective $\square \diamond F$ with positive probability. The structure of the cLTS we construct is similar to the one of the example from Fig. 2, except that the states $q_{1}$ and $q_{2}$ are replaced with two copies of the POMDP. Consistently $a$ and $\bar{a}$ are replaced by two copies of the alphabet of the POMDP with one of them bared. From $F$ states in the first copy, with a non bared action one moves to the second one, and from any state, with bared actions, one moves back from the second copy to the first one, or moves from the first copy to $r_{0}$.

The following immediate corollary is interesting since both the existence of a strategy achieving a Büchi objective almost surely, and the existence of strategy achieving a safety objective with positive probability are decidable for POMDP [2,6].

Corollary 1. The problem whether, given a POMDP $\mathrm{M}$ with subsets of states $F$ and $I$, there exists a strategy $\pi$ with $\mathbb{P}_{\pi}(\mathrm{M} \models \square \diamond F)=1$ and $\mathbb{P}_{\pi}(\mathrm{M} \models \square I)>0$, is undecidable.

Given that the general safe active diagnosis problem is undecidable, and that belief-based strategies are not sufficient to achieve safe diagnosability, we consider now the restriction of the safe active diagnosis problem to belief-based strategies. Similarly to the case of active diagnosis, we reduce the safe active probabilistic diagnosis for belief-based-strategies to some verification question on POMDP. 
Theorem 4. The safe active probabilistic diagnosis problem restricted to beliefbased strategies is in NEXPTIME and EXPTIME-hard.

\section{Conclusion}

We studied the active diagnosis and safe active diagnosis problems for probabilistic discrete event systems, within a unifying POMDP framework. While the active diagnosis problem is EXPTIME-complete, the safe active diagnosis problem is undecidable in general, and belongs to NEXPTIME when restricted to belief-based strategies. Since the lower and upper bounds do not coincide for the latter problem, we strive to close the gap between these bounds in future work. More generally, we will investigate the safe active diagnosis problem restricted to finite-memory strategies. Another problem, closely related to diagnosability, is the predictability problem: given any observation, can we detect that the occurrence of a fault before it happens? Last, given the tight relation probabilistic diagnosis has with verification problems for POMDP, we plan to investigate further POMDP problems with multiple objectives.

\section{References}

1. Baier, C., Bertrand, N., Grösser, M.: Probabilistic $\omega$-automata. Journal of the ACM 59(1), 1-52 (2012)

2. Bertrand, N., Genest, B., Gimbert, H.: Qualitative determinacy and decidability of stochastic games with signals. In: Proceedings of LICS 2009, pp. 319-328. IEEE Computer Society (2009)

3. Cabasino, M., Giua, A., Lafortune, S., Seatzu, C.: Diagnosability analysis of unbounded Petri nets. In: Proceedings of CDC 2009, pp. 1267-1272. IEEE (2009)

4. Cassez, F., Tripakis, S.: Fault diagnosis with static and dynamic observers. Fundamenta Informaticae 88, 497-540 (2008)

5. Chanthery, E., Pencolé, Y.: Monitoring and active diagnosis for discrete-event systems. In: Proceedings of SP 2009, pp. 1545-1550. Elsevier (2009)

6. Chatterjee, K., Doyen, L., Gimbert, H., Henzinger, T.A.: Randomness for free. In: Hliněný, P., Kučera, A. (eds.) MFCS 2010. LNCS, vol. 6281, pp. 246-257. Springer, Heidelberg (2010)

7. Fabre, E., Jezequel, L.: On the construction of probabilistic diagnosers. In: Proceeeding of WODES 2010, pp. 229-234. Elsevier (2010)

8. Haar, S., Haddad, S., Melliti, T., Schwoon, S.: Optimal constructions for active diagnosis. In: Proceedings of FSTTCS 2013. LIPIcs, vol. 24, pp. 527-539. Schloss Dagstuhl - Leibniz-Zentrum für Informatik (2013)

9. Morvan, C., Pinchinat, S.: Diagnosability of pushdown systems. In: Namjoshi, K., Zeller, A., Ziv, A. (eds.) HVC 2009. LNCS, vol. 6405, pp. 21-33. Springer, Heidelberg (2011)

10. Sampath, M., Lafortune, S., Teneketzis, D.: Active diagnosis of discrete-event systems. IEEE Transactions on Automatic Control 43(7), 908-929 (1998)

11. Sampath, M., Sengupta, R., Lafortune, S., Sinnamohideen, K., Teneketzis, D.: Diagnosability of discrete-event systems. IEEE Trans. Aut. Cont. 40(9), 1555-1575 (1995) 
12. Thorsley, D., Teneketzis, D.: Diagnosability of stochastic discrete-event systems. IEEE Transactions on Automatic Control 50(4), 476-492 (2005)

13. Thorsley, D., Teneketzis, D.: Active acquisition of information for diagnosis and supervisory control of discrete-event systems. Journal of Discrete Event Dynamic Systems 17, 531-583 (2007)

14. Vardi, M.Y.: Automatic verification of probabilistic concurrent finite-state programs. In: Proceedings of FOCS 1985, pp. 327-338. IEEE Computer Society Press (1985)

15. Yoo, T.-S., Lafortune, S.: Polynomial-time verification of diagnosability of partially observed discrete-event systems. IEEE Trans. Automat. Contr. 47(9), 1491-1495 (2002) 